\title{
Genetic Control of Fertility in Streptomyces coelicolor A3(2): Plasmid Involvement in the Interconversion of UF and IF Strains
}

\author{
By A. VIVIAN \\ John Innes Institute, Colney Lane, Norwich, NOR $70 \mathrm{~F}$
}

(Accepted for publication 13 September 1971)

\begin{abstract}
SUMMARY
IF (Initial Fertility) strains of Streptomyces coelicolor A3(2) are shown to harbour a plasmid (SCP I). Loss of SCP I results in a change of fertility from IF to the previously described UF (Ultra-Fertile) type. Although UF $\times$ IF crosses yield recombinants for chromosomal markers with a frequency of the order of $10^{-5}$ of the total progeny, virtually all the progeny carrying the markers of the UF parent are converted to IF, by reinfection with SCP I. Efficient conversion of UF to IF in such crosses is associated with the development of aerial mycelium. It is inferred that SCP I is responsible for the production of a diffusible substance by IF strains, which prevents the development of aerial mycelium by UF strains.
\end{abstract}

\section{INTRODUCTION}

Wild-type A3(2) of Streptomyces coelicolor is of the Initial Fertility (IF) type (Vivian \& Hopwood, I970). IF strains were found to give rise to Ultra-Fertile (UF) strains (Hopwood, Harold, Vivian \& Ferguson, 1968, 1969) spontaneously with an appreciable frequency; from 0.03 to $0.4 \%$ of the colonies produced by plating an IF culture are UF, the variation presumably reflecting clonal fluctuations. The frequency of UF production from IF was increased up to tenfold by ultraviolet irradiation and by X-rays, but the chemical mutagen $N$-methyl$N^{\prime}$-nitro- $N$-nitrosoguanidine had no significant effect (Vivian \& Hopwood, 1970). These results implied that the change from IF to UF was unlikely to be due to a simple mutation.

Evidence will be presented in this paper that the change from IF to UF involves the loss of a plasmid, and that this change can be reversed by reinfection of the UF strain with the plasmid, by contact with an IF strain. Further experiments reported here indicate an interaction of the IF and UF fertility types in a mixed culture in relation to the morphological differentiation of the culture.

\section{METHODS}

General. Complete (CM) and minimal (MM) media and standard cultural techniques were those described by Hopwood (1967), except that all crosses were performed on CM in glass Petri dishes. For this purpose the glass lid was replaced by an aluminium lid containing a sheet of sterile filter paper. This arrangement was found to favour good growth and better sporulation than the plastic dishes used for all other purposes.

Strains. The strains, which are all mutational and recombinational derivatives of Streptomyces coelicolor A3(2) (Hopwood, 1959), are listed in Table I; some have been described in previous studies of the fertility system (Hopwood et al. 1969; Vivian \& Hopwood, 1970) and others were produced during the present work.

Ultraviolet irradiation. This was carried out as described by Harold \& Hopwood (1970). Testing of fertility by 'plate crossing'. This was done by a modification of the indirect 
selection procedure of Sermonti \& Casciano (I963) as described by Hopwood et al. (I969) and Vivian \& Hopwood (1970).

Genetic analysis. Haploid recombinant selection was done in the way described by Hopwood (1967).

\section{RESULTS}

The nature of UF $\times$ IF crosses. Mixed cultures of UF and IF strains yield selected recombinants in respect of the usual, chromosomal, markers with a frequency of the order of $\mathrm{IO}^{-5}$ of the total spores harvested from the mixed culture (Vivian \& Hopwood, 1970). For example, in the cross in Table 2, a selected class of recombinants had a frequency of $2.1 \times 10^{-5}$; approximately $33 \%$ of the spores had the markers of the UF parent and $67 \%$ those of the IF parent. However, when progeny carrying the markers of the UF parent were tested for fertility type, almost all were found to be of the IF type. Since the change of fertility from UF to IF for the parentally marked (hisAI uraAI strAI) class of segregants occurred with a frequency very much (10,000 times) higher than the frequency of recombinants for chromosomal markers, the factor responsible for the difference in fertility between UF and IF strains must have been inherited independently of the chromosome.

The strains derived from UF strains by contact with IF strains as just described behaved in every way like typical IF strains. In particular they gave rise to UF strains spontaneously, or, after ultraviolet irradiation, at the normal frequencies (Vivian \& Hopwood, 1970) (Table 3). They also converted UF strains to IF as efficiently as did type IF strains.

Conversion of UF to IF in mixed culture in relation to colonial differentiation. The growth and development of Streptomyces coelicolor is complex for a prokaryote (Hopwood, 1960; Wildermuth, 1970; Wildermuth \& Hopwood, 1970). There are three main stages in the development of a colony. Substrate mycelium first develops at, and just below, the agar surface giving a culture which is flat and shiny in appearance. Later aerial mycelium grows upwards from the substrate mycelium and is recognized by its 'hairy', white appearance. Finally spores are formed by septation of the aerial mycelium and the culture surface then becomes grey (Hopwood, Wildermuth \& Palmer, 1970). The time scale of development varies with growth conditions (Wildermuth, 1970); in the present experiments aerial mycelium began to appear at about $24 \mathrm{~h}$. and increased up to about $50 \mathrm{~h}$., after which spore production gradually increased, being clearly visible to the naked eye at $72 \mathrm{~h}$.

Replicate crosses of strain I 190 hisAI uraAI strAI UF with strain I 2 pheAI IF were incu-

Table I. Characteristics of strains

\begin{tabular}{|c|c|c|c|c|c|c|c|}
\hline \multirow[b]{2}{*}{ Strain } & \multicolumn{6}{|c|}{ Relevant phenotype } & \multirow[b]{2}{*}{$\dagger$ Fertility } \\
\hline & $\arg A I$ & $\operatorname{pro} A I$ & hisAI & uraAI & pheAI & $\operatorname{str} A x$ & \\
\hline $\mathrm{A} 3(2) *$ & + & + & + & + & + & $\mathbf{S}$ & IF \\
\hline 12 & + & + & + & + & - & $\mathbf{S}$ & IF \\
\hline 1098 & + & + & + & + & - & $\mathbf{S}$ & UF \\
\hline I I4 I & + & + & + & + & - & $\mathbf{S}$ & NF \\
\hline 104 & + & + & - & - & + & $\mathbf{R}$ & IF \\
\hline I 190 & + & + & - & - & + & $\mathbf{R}$ & UF \\
\hline A506 & + & + & - & - & + & $\mathbf{R}$ & IF \\
\hline A303 & + & + & - & - & + & $\mathbf{R}$ & NF \\
\hline A427 & + & + & - & + & + & $\mathbf{S}$ & IF \\
\hline A450 & + & + & - & + & + & $\mathbf{S}$ & UF \\
\hline I 100 & - & - & + & + & - & $\mathbf{S}$ & NF \\
\hline
\end{tabular}



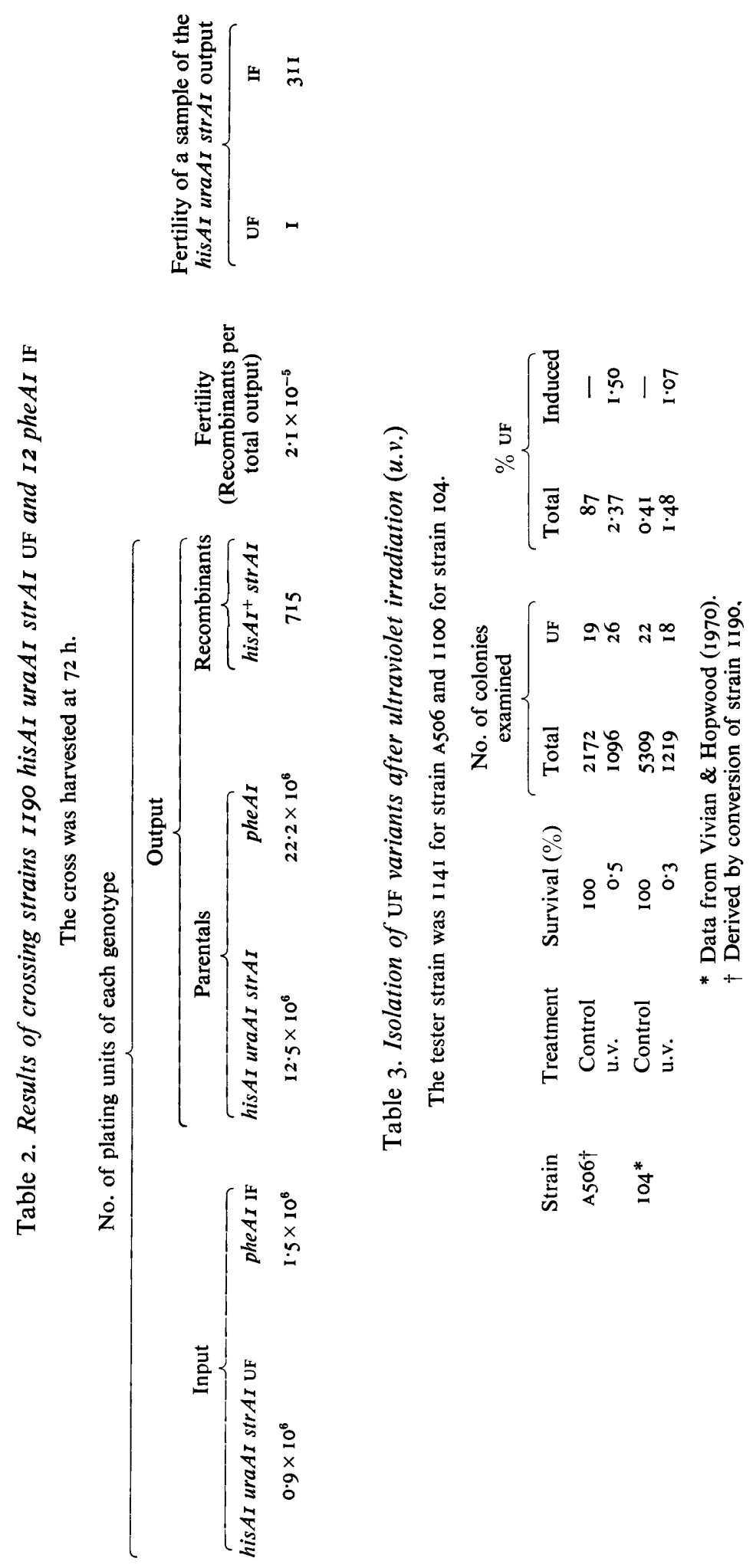


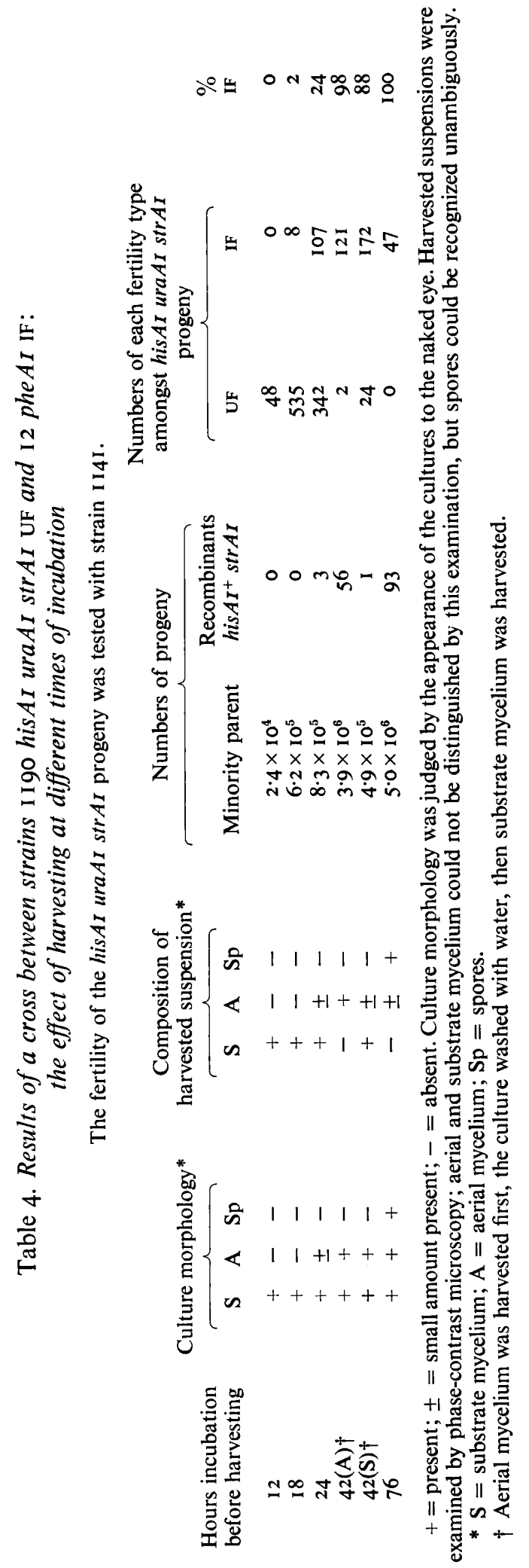


bated for varying time intervals before harvesting (Table 4). At each time of harvest, the suspensions were plated on media selecting each of the two parental marker combinations, and on a medium selecting his $\mathrm{II}^{+}$strAI recombinants. The fertility of a sample of hisAI uraAI strAI progeny was also determined.

In crosses harvested at 12 and $\mathrm{I} 8 \mathrm{~h}$. the great majority of the hisAI uraAI strAI progeny remained unchanged in fertility type: that is they were UF. These crosses had not developed aerial mycelium. At $24 \mathrm{~h}$. when aerial mycelium had begun to appear, $24 \%$ of the hisAI uraAI strAI plating units gave rise to IF colonies. At $42 \mathrm{~h}$. an attempt was made to analyse substrate and aerial mycelium separately. The aerial mycelium was harvested by gentle scraping and plated, and the substrate mycelium was then harvested, after thoroughly washing the previously scraped culture surface. From the aerial mycelium only $2 \%$ of the hisAI uraAI strAI plating units gave UF colonies, whereas from the substrate mycelium I $2 \%$ were UF. It should be emphasized that this experiment probably underestimated the difference between the two stages of development for two reasons. First, it was more difficult to break up the substrate mycelium, so that the plating units were larger than from the aerial mycelium and presumably any IF unit present in a clump of otherwise UF mycelium rendered the resulting colony IF. Results described later tend to support this notion. Secondly, it is unlikely that all the aerial fragments were removed before the substrate mycelium was harvested since they tend to be trapped between the substrate hyphae.

At $76 \mathrm{~h}$. all the hisAI uraAI strAI colonies were IF. These results suggested that conversion of UF to IF was associated with the production of aerial mycelium, and that the underlying substrate mycelium of the UF parent probably remained at least partly (if not largely) UF.

Addition of water to the Petri dish containing a developing culture, still at the substrate mycelial stage, prevented the development of aerial mycelium although vegetative growth continued. When the water was removed aerial mycelium appeared. In this way it was possible to separate the effects of culture age and colonial differentiation.

Table $5(a)$ shows the results of an experiment with strains II 90 UF and I2 IF. At $42 \mathrm{~h}$. in the absence of water, aerial mycelium was produced and nearly all the hisAI uraAI strAI progeny were converted to IF, confirming the results of the previous experiments. However, when water was added at $I 7 \mathrm{~h}$., when only substrate mycelium was visible, only $3 \%$ of the hisAI uraAI strAI progeny harvested at $42 \mathrm{~h}$. were IF. Removal of the water at $42 \mathrm{~h}$. and reincubation was followed by aerial mycelium formation at about $90 \mathrm{~h}$. At this time virtually all the hisAI uraAI strAI progeny were IF.

The experiment was repeated to see whether addition of water at times later than $17 \mathrm{~h}$. could determine a time at which the culture might have become 'committed' to aerial mycelium formation and/or to conversion of fertility type. All the crosses were harvested at $42 \mathrm{~h}$. after the addition of water at times varying from 17 to $28 \mathrm{~h}$. Even when water was added as early as $\mathrm{I} 7 \mathrm{~h}$. a small proportion ( $2 \%$ ) of If colonies was produced (Table $5 b$ ). As the time allowed for growth before the addition of water was lengthened so the proportion of If progeny amongst the hisAI uraAI strAI class increased up to $30 \%$ at $28 \mathrm{~h}$., when the culture was beginning to show obvious signs of aerial mycelium.

These results, while not defining a precise time of 'commitment' to IF conversion, clearly establish an association of conversion with aerial mycelium initiation, rather than with the absolute age of the mixed culture.

The effect of varying the input ratio of the two parental strains on the conversion of UF to IF. Experiments were done to see how much the proportion of IF in a mixed culture with UF 


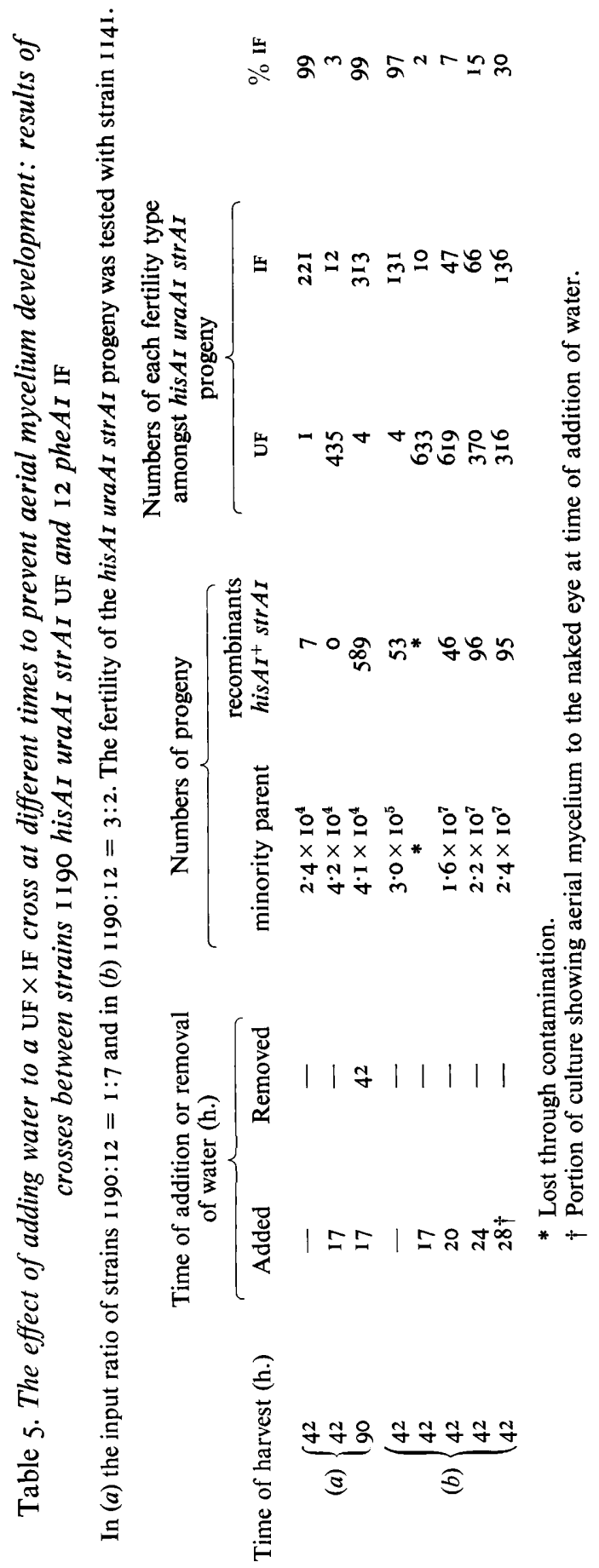




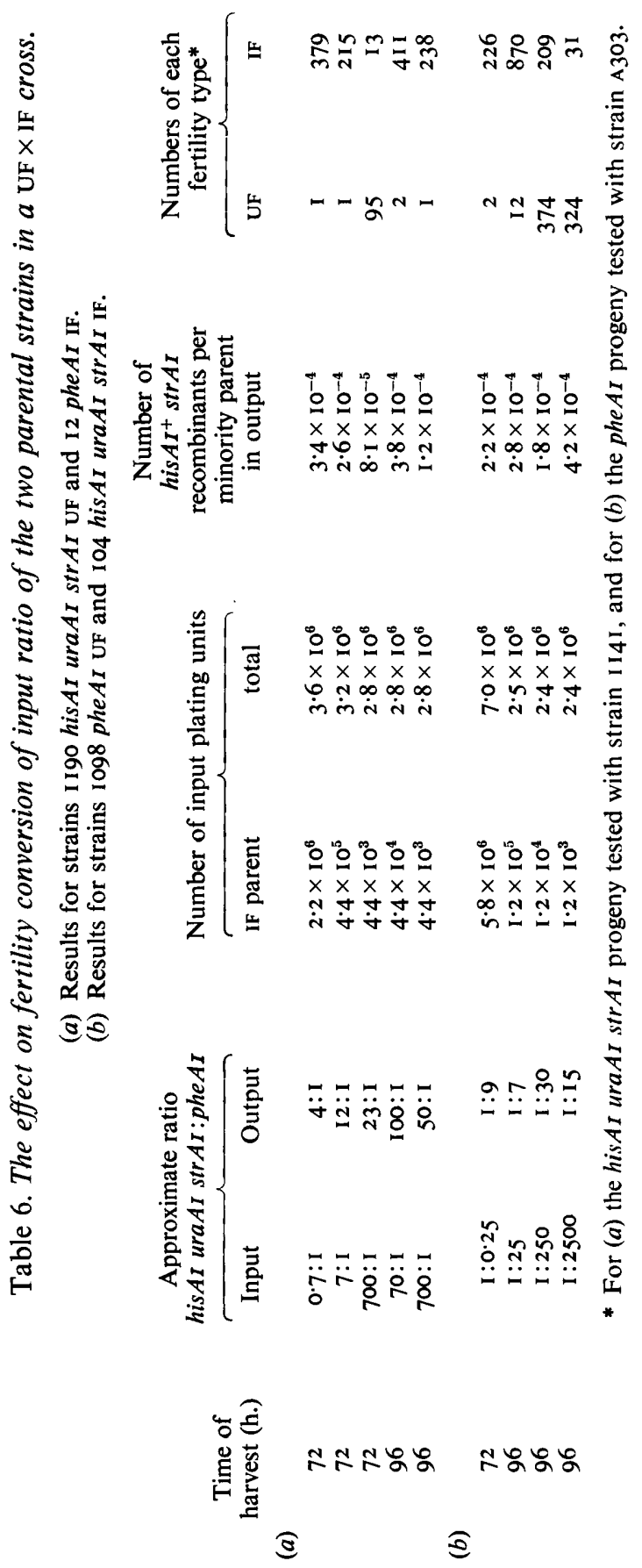


could be reduced, while still allowing efficient conversion of the UF parent to IF. Table $6(a)$ shows results obtained for strains I 190 his $A I$ uraAI strAI UF $\times$ I 2 phe AI IF and Table $6(b)$ for strains 1098 pheAI UF $\times$ I04 hisAI uraAI strAI IF. The purpose of crosses involving the same fertility types in reciprocal coupling with the markers was to test for gross distortion of output ratios by marker effects; the results suggest that the markers did not greatly affect the output ratios of the crosses. In both crosses, for approximately equal input ratios the UF marker combination tended to increase relative to the IF combination in the output, whereas for the most unequal input ratios there was a strong tendency to reduce the excess input of the UF marker combination.

The results indicate that at input ratios of UF:IF up to about 25:I in cross $(b)$ and even higher in cross $(a)$, conversion of the UF parental class was close to $100 \%$. Table $6(a)$ (lines 3 and 5) indicate that a majority of UF at $72 \mathrm{~h}$. became a majority of IF when incubated for a further $24 \mathrm{~h}$., even when the input ratio of UF to IF was as high as 700: I.

It should be emphasized that the absolute age of the various crosses was a poor indicator of their physiological age. Crosses in which the IF parent was in a small minority took longer to reach a state of aerial development comparable with the cross involving equal inputs of parental types. This was probably not due to variation in the total number of spores used to make the crosses because efforts were made to minimize such differences by adjusting the total concentrations of the mixed suspensions. The total spore input in the crosses never varied more than threefold (column 5, Table 6).

A possible conclusion from the results of Table 6 is that UF is at a disadvantage in producing aerial mycelium when in mixed culture with IF, since a small minority of IF effectively 'suppressed' aerial mycelium production in a large majority of UF input. This possibility was tested directly in experiments described in the next section. Such an effect could also explain the very efficient conversion of UF to IF in the aerial mycelium of a mixed culture, but not in the substrate mycelium, described earlier; 'converted' (IF) substrate mycelium would be able to proceed to aerial mycelium development, while unconverted (UF) would not.

The differential ability of UF and IF components in a mixed culture to produce aerial mycelium. An observation suggesting that UF mycelium may be prevented by IF from initiating the aerial phase was the following. When a plate of colonies, some IF and some UF, was crossed by replica plating to a lawn of a UF tester strain, the two types of colony could be distinguished by their effect upon the growth of the UF tester strain (Fig. I): the IF colonies produced a halo in the UF background where no aerial mycelium developed, whereas the UF colonies did not. The fertility of the two types of colony, as described previously (Vivian \& Hopwood, I970), was similarly low with the UF tester, but markedly different with an NF (Normal Fertility) tester (Fig. I c).

Figure 2 shows the results of a more direct investigation of this phenomenon. UF or IF strains carrying the marker hisAI were grown in the centre of a Petri dish and replicated to a lawn of either a UF or an IF strain carrying the markers hisAI uraAI strAI. Thus, at the centre of each dish there was a mixed culture of IF $\times$ IF, IF $\times$ UF or UF $\times$ UF surrounded respectively by IF, UF or UF growth.

The mixed IF on UF culture (dish 2) is surrounded by a zone where the UF culture did not produce aerial mycelium. Absence of this zone around homologous UF on UF (dish I) and IF on IF (dish 3) crosses indicates that it was not due to a starvation effect caused by the increased inoculum in the mixed region, nor to an effect of the markers in the strains.

Petri dishes 4 to 9 in Fig. 2 are replicas of their respective crosses (dishes $I$ to 3 ) on media which allow distinction of the two parental marker combinations. Clearly there was no migration of the IF markers beyond the mixed zone. 


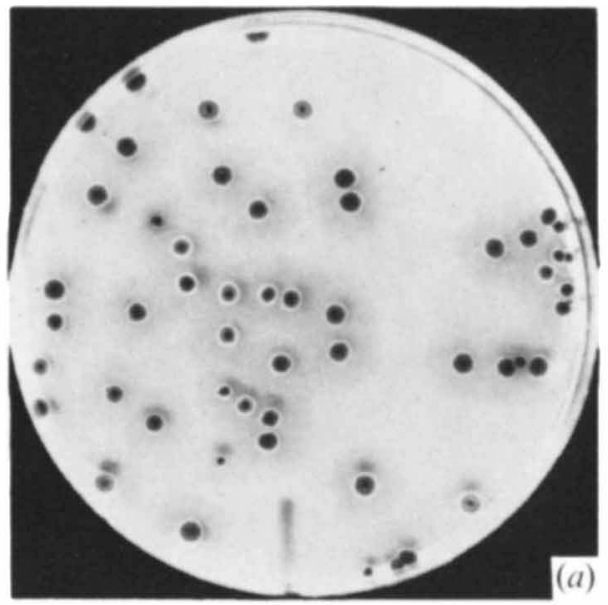

Original colonies

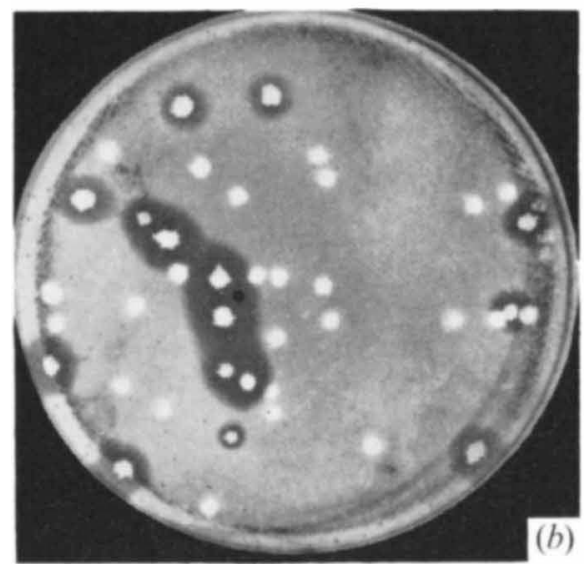

Plate cross 1 , tester

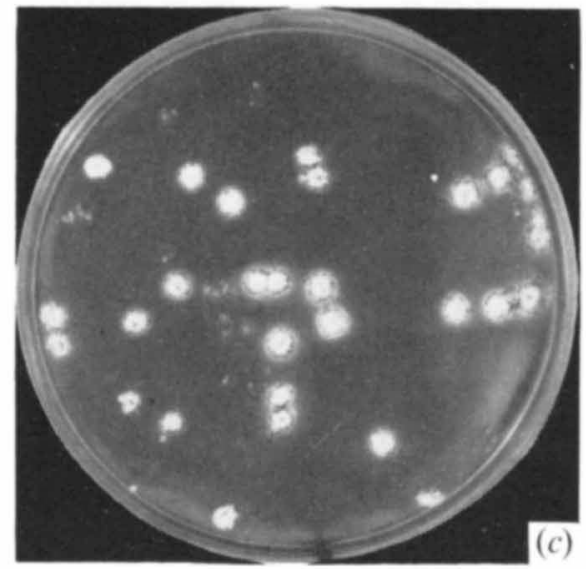

Replica from $\backslash$ plate cross

Fig. I (a) hisAI uraAI strAI progeny from a mixed culture of II90 hisAI uraAI strAI UF and 12 phe $A I$ IF. (b) Plate cross on CM prepared by replicating plate $(a)$ to a lawn of tester strain 1098 phe AI UF. IF colonies on the UF background are surrounded by a dark zone where aerial mycelium development of the UF tester strain has been prevented; UF colonies on the same plate are not. The UF and IF colonies are distinguished by plate $(c)$. The colonies shown in $(a)$ were plate crossed to tester strain II4I pheAI NF. This plate cross (not shown) was then replicated to the plate of selective medium in $(c)$ which distinguishes the colonies on the basis of their fertility with the NF tester. UF colonies give many recombinants; IF colonies give few or no recombinants under these conditions.

Petri dishes I to 3 were also replicated to a suitably marked NF tester strain, I I 4 I phe AI which allowed the fertility of both the parental types to be tested. The plate crosses so produced (which are not shown in Fig. 2) were then replicated to a medium selecting his $\mathrm{AI}^{+}$ phe $\mathrm{AI}^{+}$recombinants. The result is shown in Fig. 2, dishes Io to I2. As expected, UF on UF gave a high frequency of recombinants over the whole plate (dish I0) whereas IF on IF gave a low level over the whole plate (dish I2). The cross of IF on UF (dish II) showed an inner zone where the mixed IF + UF inoculum gave a low level of recombination consistent with its 
UF on $\mathrm{U}$ !

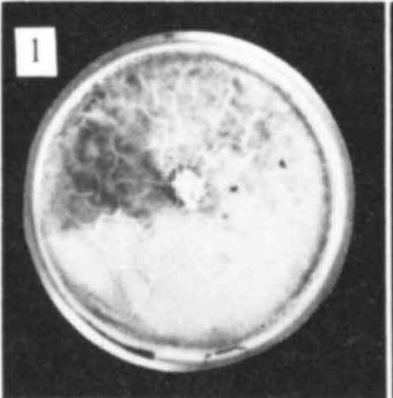

Plate cross on $\mathrm{CM}$

Parental selection histidine

Parental selection: histidine

+ uracil

+streptomycin

Recombinant selection from test with NF uracil
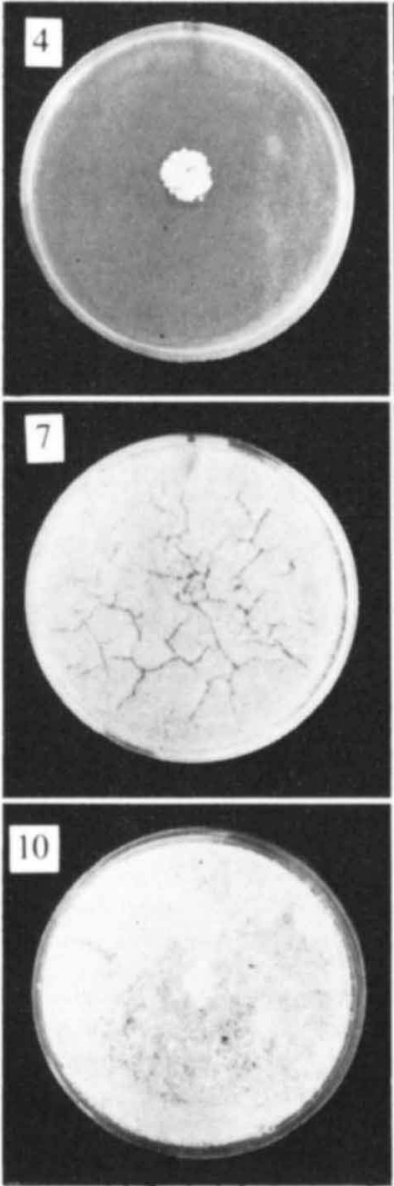

If on UF
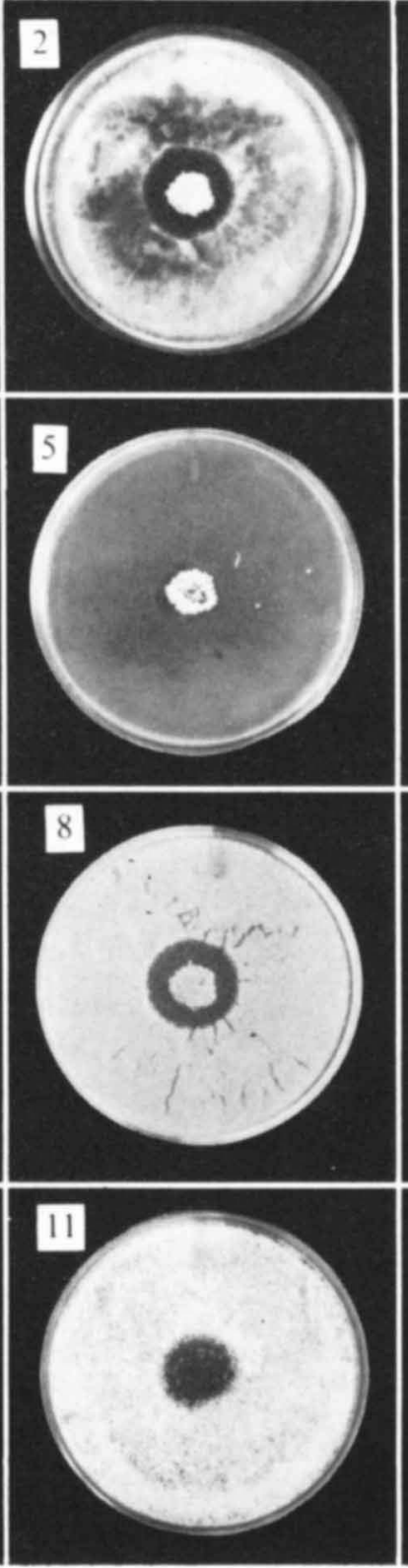

If on If
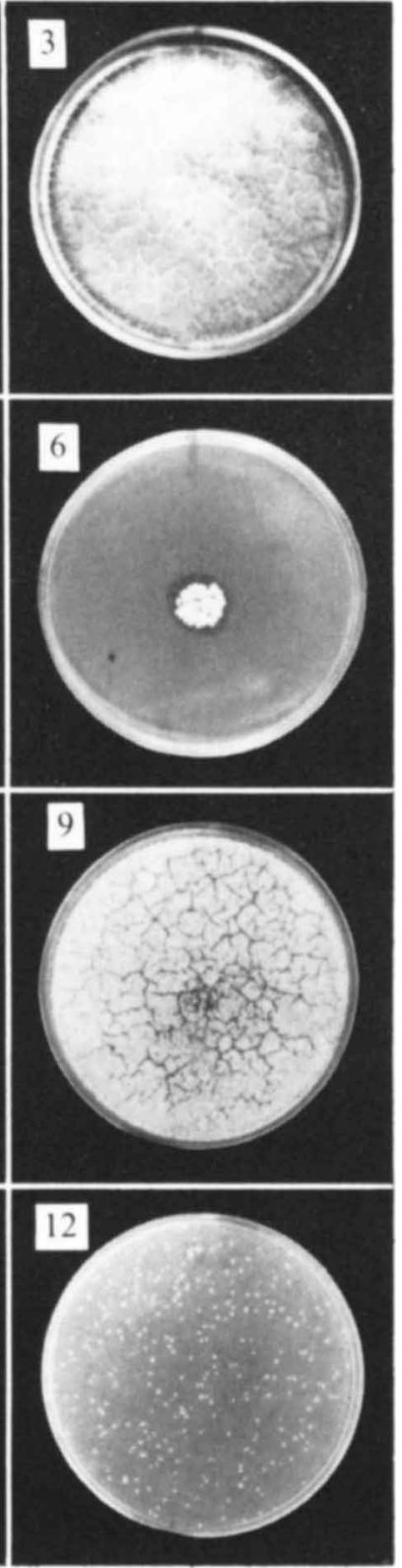

having become entirely IF by conversion of the UF parent. Outside of this area, the region devoid of aerial mycelium on the original plate (dish 2 ) did not replicate and hence gave no recombinants. Beyond this zone the fertility of the UF parent remained high, indicating that there was no migration of IF fertility outwards through the UF parent.

A small piece of substrate mycelium from the region devoid of aerial mycelium in dish 2 was picked to a fresh plate of $\mathrm{CM}$ and streaked out. Colonies of normal appearance 
developed with aerial mycelium and spores. These were tested for fertility and genotype and found to be hisAI ura AI strAI UF, showing that the UF strain in the region where aerial mycelium development had been inhibited, had not undergone a permanent change.

\section{DISCUSSION}

The frequency of UF production from IF strains is higher than that expected for a chromosomal mutation (Hopwood et al. 1968, 1969; Vivian \& Hopwood, 1970) but is compatible with its being due to loss of a plasmid. The finding that IF strains can 'convert' UF strains to IF with a frequency almost $10,000-$ fold higher than the frequency of inheritance of chromosomal markers, and that such 'converted' strains are as stable as type IF strains, clearly demonstrates that the change from UF to IF involves the acquisition of an extra-chromosomal element: by definition, a plasmid. It is proposed to call the plasmid SCP I (Streptomyces coelicolor plasmid I).

It seems that SCP I is responsible either directly or indirectly for the production of a diffusible substance which prevents or reduces the initiation of aerial mycelium by cultures lacking SCP I. Thus IF itself, or an intimate IF + UF mixture, prevents the development of UF aerial mycelium. It is possible that production of this developmental inhibitor has to be induced by the presence of a strain lacking SCP I.

In a UF $\times$ IF cross, the IF strain, which harbours SCP I, is able to produce aerial mycelium and thereafter spores, whereas any part of the culture which lacks the plasmid (and so remains UF) is prevented by the IF from developing aerial mycelium, and hence spores. It is possible that the plasmid is transferred to a minority of the UF substrate mycelium, as indicated by the results of harvesting a UF $\times$ IF cross at times close to aerial mycelium initiation ( $8 \mathrm{~h}$.) (Table 4). The dramatic increase in the proportion of UF converted to IF is clearly associated with the development of aerial mycelium and it is not necessary to postulate a progressive conversion of the underlying substrate mycelium. The end result, as judged by the analysis of spores from the mixed culture, is almost total conversion of the UF parent to IF. The results obtained in Table 5 discount the possibility that age of the culture per se is responsible for the efficient conversion of UF to IF.

Differences previously observed between UF and IF strains centred on their respective behaviours when crossed with NF strains. The most obvious difference was that UF strains were more fertile (indeed 'ultra-fertile') with NF than were IF strains: the frequency of

Fig. 2. Suppression by IF of aerial mycelium production by a UF strain: for full explanation see text.

I to 3. Plate crosses on CM. (I) A central patch of A450 hisAI UF was replica plated on to a lawn of I 190 hisAI uraAI strAI UF. (2) As (I) but A427 hisAI IF on a lawn of I 190 hisAI uraAI strAI UF. As (I) but A427 hisAI IF on a lawn of 104 hisAI uraAI strAI IF.

4 to 6 . Replicas from plate crosses $\mathrm{I}$ to 3 respectively on medium selecting for his $A I$ parental phenotype.

7 to 9. Replicas from plate crosses $\mathrm{I}$ to 3 respectively on medium selecting for his AI uraAI strAI parental phenotype.

Io to I2. Plate crosses I to 3 were replica plated to further plate crosses (not shown here) with I 4 I phe AI NF as tester strain. Io to 12 are the respective replicas from these plate crosses on medium selective for recombinants. (I0) The level of recombination with NF is high, confirming that the growth on $I$ is all UF. (II) The inner circular area gives few recombinants, showing that it has become uniformly IF. Outside of this is a ring where aerial mycelium development has been prevented; this region failed to replicate and so gave no recombinants. Around this the culture remains UF and gives many recombinants. (12) The level of recombination with NF is low, confirming that the growth on 2 is all IF. 
recombinants in a UF $\times$ NF cross reaches $100 \%$ (Hopwood et al. 1968, 1969), whereas in an IF $\times$ NF cross it is around 10\% (Vivian \& Hopwood, I970). The increased fertility of UF $\times$ NF as compared with IF $\times$ NF crosses can be visualized as due to the abolition of asexual reproduction in the former, but not in the latter. It is a reasonable hypothesis that this is due to SCP I harboured by the NF strain. Because of the ultra-fertile nature of UF $\times$ NF crosses it has not been possible to demonstrate conversion of UF by NF independently of the transfer of chromosomal markers. In other words, if NF does harbour SCP I, its inheritance must be associated with that of chromosomal markers since all recombinant progeny from $\mathrm{UF} \times \mathrm{NF}$ crosses are NF (Hopwood et al. 1969).

It should be emphasized that loss or gain of SCP $\mathrm{I}$ is not associated with a change from donor to recipient or vice versa, since both IF and UF strains behave as recipients in crosses with NF strains. The difference between donor and recipient behaviour has been located chromosomally by crossing NF and IF strains (Vivian \& Hopwood, 1970). Thus, although the implication of a plasmid in the control of sexual reproduction in Streptomyces coelicolor is in itself reminiscent of the situation in eubacteria like Escherichia coli (Hayes, 1968), there are at present no close analogies between the two systems. On the contrary, the association between a fertility factor and colonial differentiation described in this paper, promises to illuminate problems which are beyond the scope of studies with the simple eubacteria.

It is a pleasure to thank Professor D. A. Hopwood for many helpful discussions during the course of this work and also for his advice and criticism during the preparation of this paper. I am also indebted to Dr K. F. Chater for suggesting the experiment shown in Table 5 . I would particularly like to thank Mrs Helen Tovell for her skilled assistance throughout the work.

I am grateful to the Science Research Council for a post-doctoral stipend from grant number B/SR/4060 to D. A. Hopwood.

\section{REFERENCES}

Harold, R. J. \& Hopwood, D. A. (1970). Ultraviolet-sensitive mutants of Streptomyces coelicolor. I. Phenotypic characterization. Mutation Research ro, 427-438.

HAYEs, W. (1968). The Genetics of Bacteria and Their Viruses. Oxford: Blackwell.

Hopwood, D. A. (1959). Linkage and the mechanism of recombination in Streptomyces coelicolor. Annals of the New York Academy of Sciences 81, 887-898.

Hopwood, D. A. (1960). Phase-contrast observations on Streptomyces coelicolor. Journal of General Microbiology 22, 295-302.

Hopwood, D. A. (1967). Genetic analysis and genome structure in Streptomyces coelicolor. Bacteriological Reviews 31, 373-403.

Hopwood, D. A., Harold, R. J., Vivian, A. \& Ferguson, H. M. (1968). Non-selective genetic analysis in Streptomyces coelicolor. Heredity 23, 628.

Hopwood, D. A., Harold, R. J., Vivian, A. \& Ferguson, H. M. (1969). A new kind of fertility variant in Streptomyces coelicolor. Genetics 62, 46I-477.

Hopwood, D. A., Wildermuth, H. \& Palmer, H. M. (1970). Mutants of Streptomyces coelicolor defective in sporulation. Journal of General Microbiology 6r, 397-408.

Sermonti, G. \& Casciano, S. (1963). Sexual polarity in Streptomyces coelicolor. Journal of General Microbiology 33, 293-30I.

Vivian, A. \& Hopwood, D. A. (1970). Genetic control of fertility in Streptomyces coelicolor A3(2): the IF fertility type. Journal of General Microbiology 64, I0I-I 17.

Wildermuth, H. (1970). Development and organization of the aerial mycelium in Streptomyces coelicolor. Journal of General Microbiology 60, 43-50.

Wildermuth, H. \& HopwoOD, D. A. (1970). Septation during sporulation in Streptomyces coelicolor. Journal of General Microbiology 60, $5 \mathrm{I}-59$. 